Ciência eNatura, Santa Maria, v. 37 Part 1 , p. 462-466

\title{
Some fixed point theorems for Kannan mapping in the modular spaces
}

\author{
S. J. Hosseini Ghoncheh \\ Department of Mathematics, College of Science, Takestan Branch, Islamic Azad University, Takestan, \\ Iran. sjhghoncheh@gmail.com, sjhghoncheh@tiau.ac.ir
}

\begin{abstract}
In this article, a new version of Kannan mapping theorem in modular space is presented. The main result of this paper is the existence of fixed point of Kannan mapping in complete modular spaces that have Fatou property.
\end{abstract}

Key words: Fixed point, Kannan map, Modular space.

AMS subject classification: 47H10, 46A19, 46B20, 47H09. 


\section{Introduction}

In this article, existence of a fixed point of Kannan mapping in the modular space is proved. The theory of this space was initiated by Nakano [9] in 1950 in connection with the theory of order spaces and redefined and generalized by Musielak and Orlicz [8] in 1959. In order to do this and for the sake of convenience, some definitions and notations are recalled from [2],[3], [4], [5], [7], [8], [9] and [10].

Definition 1.1 Let $X$ be an arbitrary vector space over $K(=\mathrm{R}$ or $\mathrm{C})$.

A functional $\rho: X \rightarrow[0,+\infty)$ is called modular if:

1. $\rho(x)=0$ if and only if $x=0$.

2. $\rho(\alpha x)=\rho(x)$ for $\alpha \in \mathrm{K}$ with $|\alpha|=1 ; \forall x, y \in$ $X$.

3. $\rho(\alpha x+\beta y) \leq \rho(x)+\rho(y)$ if $\alpha, \beta \geq 0, \alpha+$ $\beta=1 ; \forall x, y \in X$.

Definition 1.2 If 3 in Definition 1.1 is replaced by:

$\rho(\alpha x+\beta y) \leq \alpha^{s} \rho(x)+\beta^{s} \rho(y)$ for $\alpha, \beta \geq$ $0, \alpha^{s}+\beta^{s}=1$ with an $s \in(0,1]$

then the modular $\rho$ is called an $s$-convex modular; and if $s=1, \rho$ is called a convex modular.

Definition 1.3 A modular $\rho$ defines a corresponding modular space, i.e. the space $X_{\rho}$ is given by

$X_{\rho}=\{x \in X \mid \rho(\gamma x) \rightarrow 0$ as $\gamma \rightarrow 0\}$.

Definition 1.4 Let $X_{\rho}$ be a modular space.

1. A sequence $\left\{x_{n}\right\}$ in $X_{\rho}$ is said to be:

(a) $\rho$-convergent to $x$ if $\rho(x n-x) \rightarrow 0$ as $n \rightarrow+\infty$. (b) $\rho$-Cauchy if $\rho(x n-x m)) \rightarrow 0$ as $n, m \rightarrow+\infty$.

2. $X_{\rho}$ is $\rho$-complete if any $\rho$-Cauchy sequence is $\rho$-convergent.

3. A subset $B C X_{-} \rho$ is said to be $\rho$-closed if for any sequence $\left\{x_{n}\right\} C B$

with $x_{n} \rightarrow x$, then $x \in B . \overline{B^{\rho}}$ denotes the closure of $B$ in the sense of $\rho$.

4. A subset $B C X \rho$ is called $\rho$-bounded if:

$$
\delta_{\rho}(B)=\sup _{x, y \in B} \rho(x-y)<+\infty,
$$

where $\delta \rho(B)$ is called the $\rho$-diameter of $B$.

5. We say that $\rho$ has Fatou property if:

$$
\rho(x-y) \leq \liminf \rho(x n-y n)
$$

whenever

$$
x_{n} \stackrel{\rho}{\rightarrow} x, \quad y_{n} \stackrel{\rho}{\rightarrow} y
$$

6. $\rho$ is said to satisfy the $\Delta 2$-condition if $: \rho(2 x n) \rightarrow 0$ as $n \rightarrow+\infty$,

whenever $\rho(x n) \rightarrow 0$ as $n \rightarrow+\infty$.

Now, we state the definition of Kannan mapping in the modular space (see [6]).

Definition 1.5 $T: X \rho \rightarrow X \rho$ is called a Kannan mapping, if there exist

$$
\begin{aligned}
& c \geq 1 \text { and } \alpha \in\left[0, \frac{1}{2}\right) \text {, such that } \\
& \begin{aligned}
\rho(c(T x-T y)) & \leq \alpha(\rho(T x-x)+\rho(T y \\
& -y))
\end{aligned} \\
& \text { for all } x, y \in X \rho \text {. }
\end{aligned}
$$

Theorem 1.6 Let X $\rho$ be complete modular space which $\rho$ satisfies the Fatou property. Suppose $T$ : $X \rho \rightarrow X \rho$ be a Kannan mapping and assume that there exists $x \in X \rho$ which $\rho(T x-x)<\infty$. Then $T$ has a unique fixed point. 
Proof. By induction on $n \in \mathrm{N}$, we prove the following inequality,

$$
\rho\left(c\left(T^{n+1} x-T^{n} x\right)\right) \leq\left(\frac{\alpha}{1-\alpha}\right)^{n} \rho(T x-x) .
$$

Let $x \in X_{-} \rho$, for $n=1$ inequality holds.

Assume for $n=k \in \in \mathrm{N}$ we have

$$
\rho\left(c\left(T^{k+1} x-T^{k} x\right)\right) \leq\left(\frac{\alpha}{1-\alpha}\right)^{k} \rho(T x-x) .
$$

Now, for $n=k+1$,

$$
\begin{aligned}
& \rho\left(c\left(T^{k+2} x-T^{k+1} x\right)\right) \\
& \quad \leq \alpha\left(\rho\left(T^{k+2} x-T^{k+1} x\right)\right. \\
& \left.+\rho\left(T^{k+1} x-T^{k} x\right)\right)
\end{aligned}
$$

Therefore

$$
\begin{gathered}
\rho\left(c\left(T^{k+2} x-T^{k+1} x\right)\right)-\alpha \rho\left(T^{k+2} x-T^{k+1} x\right) \\
\leq \alpha \rho\left(T^{k+1} x-T^{k} x\right) \\
\leq \frac{\alpha^{k+1}}{(1-\alpha)^{k}} \rho(T x-x),
\end{gathered}
$$

and since

$$
\begin{aligned}
(1-\alpha) \rho\left(c \left(T^{k+2}\right.\right. & \left.\left.x-T^{k+1} x\right)\right) \\
& \leq \rho\left(c\left(T^{k+2} x-T^{k+1} x\right)\right) \\
& -\alpha \rho\left(T^{k+2} x-T^{k+1} x\right)
\end{aligned}
$$

Therefore

$$
\begin{aligned}
(1-\alpha) \rho\left(c \left(T^{k+2} x\right.\right. & \left.\left.-T^{k+1} x\right)\right) \\
& \leq \frac{\alpha^{k+1}}{(1-\alpha)^{k}} \rho(T x-x),
\end{aligned}
$$

and this shows that

$$
\rho\left(c\left(T^{k+2} x-T^{k+1} x\right)\right) \leq \frac{\alpha^{k+1}}{(1-\alpha)^{k+1}} \rho(T x-x) .
$$

Now, let $m>n$, then we have

$$
\begin{gathered}
\rho\left(c\left(T^{n} x-T^{m} x\right)\right) \\
\leq \alpha\left(\rho\left(T^{n} x-T^{n-1} x\right)\right. \\
\left.+\rho\left(T^{m} x-T^{m-1} x\right)\right) \\
\leq \alpha\left(\left(\frac{\alpha}{1-\alpha}\right)^{n-1} \rho(T x-x)+\left(\frac{\alpha}{1-\alpha}\right)^{m-1} \rho(T x-x)\right),
\end{gathered}
$$

Letting $n$ tend to infinity, then

$$
\lim _{n \rightarrow \infty} \rho\left(c\left(T^{n} x-T^{m} x\right)\right)=0
$$

Consequently, $\left\{T^{n} x\right\}$ is a Cauchy sequence. Then by completeness of $X \rho$, there is a $z \in X \rho$ such that $\operatorname{Tn} x \rightarrow z$. Now, we show that $T z=$ $z$. By Fatou property we have

$$
\rho(T z-z) \leq \liminf _{n} \rho\left(T z-T^{n} z\right)
$$

Since

$$
\rho\left(T z-T^{n} z\right) \leq \rho\left(c\left(T z-T^{n} z\right)\right)
$$

we obtain

$\liminf _{n} \rho\left(T z-T^{n}(x)\right) \leq \liminf _{n} \rho\left(c\left(T z-T^{n} x\right)\right)$

$\liminf _{n} \alpha\left(\rho(T z-z)+\rho\left(T^{n} x-T^{n-1} x\right)\right) \leq$

then

$(1-\alpha) \rho(T z-z) \leq \liminf _{n} \alpha\left(\rho\left(T^{n} x-T^{n-1} x\right)\right)$

If $n$ tends to infinity we have $T z=z$. Thus $z$ is a fixed point of $T$ and $z$ is unique.

Otherwise, if $u \in X \rho$ that $=u, u \neq z$ then

$$
\rho(z-u)=\rho(T z-T u)
$$

$\rho(T u-u))$

$$
\leq \alpha(\rho(T z-z)+
$$

$$
=0
$$

therefore

$$
\rho(z-u)=0
$$

and this means that

$$
z=u \text {. }
$$

Definition 1.7 Let $T$ be a map of $X$ into itself, let $z \in X$. T is $\rho$-sequentially continuous at $z$, if and only if when $x n \rightarrow z$, then $T(x n) \rightarrow$ $T(z)$.

Theorem 1.8 Let $X \rho$ be a modular space which satisfies $\Delta 2$-condition. Let $T$ be a map of $X$ into itself and there exists a $c \geq 1$ such that:

(1) $\quad \rho(c(T x-T y)) \leq \alpha(\rho(x-T x)+\rho(y-$ Ty)); for $0<\alpha<\frac{1}{2} ; x, y \in X$.

(2) $T$ is sequentially continuous at a point $u \in X$. 
(3) There exists a point $x \in X$ such that the sequence of iterates $\left\{T^{n} x\right\}$ has a subsequence $\left\{T^{n_{i}} x\right\}$ converging to $u$.

Then $u$ is the unique fixed point of $T$.

Proof. $T$ is sequentially continuous at $u$, therefore we have $T^{n_{i}+1} x$ converges to $T u$.

By $\Delta 2$-condition we have

$$
\rho\left(c\left(T^{n_{i}} x-u\right)\right) \rightarrow 0 ; \rho\left(c\left(T^{n_{i}+1} x-T u\right)\right) \rightarrow 0 .
$$

Suppose $u \neq T u$.

Let

$$
0<\epsilon<\frac{1}{3}\left(\rho\left(\frac{1}{4}(c(u-T u))\right),\right.
$$

then there exists a positive integer $N$, such that for $n_{i}>N$,

$$
\begin{aligned}
\rho\left(c\left(T^{n_{i}} x-u\right)\right) & <\epsilon \text { and } \rho\left(c\left(T^{n_{i}+1} x-T u\right)\right) \\
& <\epsilon .
\end{aligned}
$$

Now, we claim that

$$
\rho\left(c\left(T^{n_{i}} x-T^{n_{i}+1} x\right)\right)>\epsilon ; n_{i}>N
$$

Because if

$\rho\left(c\left(T^{n_{i}} x-T^{n_{i+1}} x\right)\right) \leq \epsilon$

then

$$
\begin{gathered}
\rho\left(\frac{1}{4}(c(u-T u))\right) \leq \rho\left(c\left(u-T^{n_{i}} x\right)\right)+ \\
\rho\left(c\left(T^{n_{i}} x-T^{n_{i}+1} x\right)\right)+\rho\left(c\left(T^{n_{i}+1} x-T u\right)\right) \\
<3 \epsilon
\end{gathered}
$$

and this is a contradiction.

On the other hand,

$$
\begin{aligned}
& \rho\left(c\left(T^{n_{i}+1} x-T^{n_{i}+2} x\right)\right) \\
& \leq \alpha\left(\rho\left(T^{n_{i}} x-T^{n_{i}+1} x\right)\right. \\
& \left.+\rho\left(T^{n_{i}+1} x-T^{n_{i}+1} x\right)\right),
\end{aligned}
$$

Therefore

$$
\begin{aligned}
& \rho\left(c\left(T^{n_{i}+1} x-T^{n_{i}+2} x\right)\right) \\
& \quad \leq \frac{\alpha}{1-\alpha} \rho\left(T^{n_{i}} x-T^{n_{i}+1} x\right)
\end{aligned}
$$

Let $n_{k}>n_{j}>N$, thus

$$
\begin{gathered}
\rho\left(c\left(T^{n_{k}} x-T^{n_{k}+1} x\right)\right) \\
\leq\left(\frac{\alpha}{1-\alpha}\right)^{n_{k}-n_{j}} \rho\left(T^{n_{j} x}\right. \\
\left.-T^{n_{j}+1} x\right)
\end{gathered}
$$

Let $n_{k}$ tends to infinity, then we have

$$
\rho\left(c\left(T^{n_{k}} x-T^{n_{k}+1} x\right)\right) \rightarrow 0
$$

this is a contradiction, therefore $T(u)=u$.

If $z \in X \rho$ be a point such that $T z=z$ then

$$
\begin{aligned}
& \rho(u-z)=\rho(T u-T z) \\
& \leq \alpha(\rho(u-T u)+\rho(z-T z)) \\
& =0
\end{aligned}
$$

Hence $z=u$, and the theorem is proved.

\section{References}

A. Ait Taleb and E. Hanebaly. A fixed point theorem and its application to integral equations in modular function Spaces. Proc. Amer. Math. Soc., 128 (2000) 419-426.

T. Dominguez Benavides, M. A. Khamsi and S. Samadi. Uniformly Lipschitzian mappings in modular function spaces. Nonlinear Anal., 46 (2001) 267\{278.

F. Khojasteh and A. Razani. fixed point theorems for Q-asymptotic con- tractions of Meir-Keeler type in modular spaces. Mathematics Scientific Journal 5 (2010) 3140.

A. Hajji and E. Hanebaly. fixed point theorem and its application to perturbed integral equations in modular function spaces. Electron. J.Differential Equations, 105 (2005) $11 \mathrm{pp}$.

E. Hanebaly. fixed point theorems in modular space. arXiv: math. FA/0511319v1, 12 Nov 2005.

R. Kannan. Some results on fixed points II. American Mathematical Monthly, 76 (1969) 405-408. 
M. A. Khamsi. Nonlinear semigroups in modular function spaces. Math. Japon., 37 (1992)291-299.

J. Musielak and W. Orlicz. On modular spaces. Studia Math., 18 (1959) 591-597.

H. Nakano. Modular semi-ordered spaces. Tokyo (1959).

A. Razani, E. Nabizadeh, M. Beygmohamadi and S. Homaeipour. fixed points of nonlinear and asymptotic contractions in the modular space. Abstract and Applied Analysis, doi:10.1155/2007/40575, (2007) 10 pages. 